\title{
Ion Transport Modulators Differentially Modulate Inflammatory Responses in THP-1-Derived Macrophages
}

\author{
Steven C. Mitini-Nkhoma ${ }^{(D)},{ }^{1,2}$ Narmada Fernando, ${ }^{1}$ G. K. D. Ishaka, ${ }^{1}$ \\ Shiroma M. Handunnetti ${ }^{D}{ }^{1}$ and Sisira L. Pathirana ${ }^{1}$ \\ ${ }^{1}$ Institute of Biochemistry, Molecular Biology and Biotechnology, University of Colombo, No. 90, Cumaratunga Munidasa Mawatha, \\ Colombo 3, Sri Lanka \\ ${ }^{2}$ Malawi-Liverpool-Wellcome Trust Clinical Research Programme, P.O. Box 30096, Chichiri, Blantyre 3, Malawi
}

Correspondence should be addressed to Steven C. Mitini-Nkhoma; smnkhoma@gmail.com

Received 24 October 2020; Revised 19 February 2021; Accepted 25 March 2021; Published 8 April 2021

Academic Editor: Enrique Ortega

Copyright (c) 2021 Steven C. Mitini-Nkhoma et al. This is an open access article distributed under the Creative Commons Attribution License, which permits unrestricted use, distribution, and reproduction in any medium, provided the original work is properly cited.

\begin{abstract}
Ion transport modulators are most commonly used to treat various noncommunicable diseases including diabetes and hypertension. They are also known to bind to receptors on various immune cells, but the immunomodulatory properties of most ion transport modulators have not been fully elucidated. We assessed the effects of thirteen FDA-approved ion transport modulators, namely, ambroxol $\mathrm{HCl}$, amiloride $\mathrm{HCl}$, diazoxide, digoxin, furosemide, hydrochlorothiazide, metformin, omeprazole, pantoprazole, phenytoin, verapamil, drug $\mathrm{X}$, and drug $\mathrm{Y}$ on superoxide production, nitric oxide production, and cytokine expression by THP-1-derived macrophages that had been stimulated with ethanol-inactivated Mycobacterium bovis BCG. Ambroxol $\mathrm{HCl}$, diazoxide, digoxin, furosemide, hydrochlorothiazide, metformin, pantoprazole, phenytoin, verapamil, and drug Y had an inhibitory effect on nitric oxide production, while all the test drugs had an inhibitory effect on superoxide production. Amiloride $\mathrm{HCl}$, diazoxide, digoxin, furosemide, phenytoin, verapamil, drug $\mathrm{X}$, and drug $\mathrm{Y}$ enhanced the expression of IL- $1 \beta$ and TNF- $\alpha$. Unlike most immunomodulatory compounds currently in clinical use, most of the test drugs inhibited some inflammatory processes while promoting others. Ion pumps and ion channels could therefore serve as targets for more selective immunomodulatory agents which do not cause overt immunosuppression.
\end{abstract}

\section{Introduction}

The use of immunomodulators has increased significantly over the last few decades, in part due to a rise in the prevalence of autoimmune diseases worldwide $[1,2]$. Corticosteroids and nonsteroidal anti-inflammatory drugs (NSAIDS) are two of the oldest and most commonly used classes of immunomodulators in clinical practice. The term "corticosteroid" encompasses various steroid hormones produced by the adrenal cortex and their synthetic analogues [3]. Corticosteroids bind to cytoplasmic steroid receptors, following which the receptor-ligand complex traverses the nuclear membrane and modulates the transcription of various genes [3]. In addition to modulating transcription, corticosteroids can also directly modulate the activity of various proteins including G-protein-coupled receptors [4]. Corticosteroids induce a wide range of physiological changes and are thus associated with numerous adverse effects including osteoporosis and Cushing's syndrome [5].

NSAIDS on the other hand have a relatively narrow activity spectrum and primarily inhibit the activity of cyclooxygenase (COX) 1 and 2 . COX1 and COX2 catalyse the production of prostaglandins, which mediate various inflammatory processes $[6,7]$. However, as prostaglandins are also involved in the protection of the gastric mucosa from gastrointestinal secretions, NSAIDS at times cause peptic ulceration [8]. More selective COX2 inhibitors are generally less likely to cause peptic ulceration but are associated with an increased risk of thrombosis [7, 9].

As the demand for immunomodulators continues to increase, there is a need for safer immunomodulatory agents. Over the last few decades, there has been increasing interest 
in the use of ion transport modulators as immunomodulatory agents. Ion transport modulators are a diverse group of compounds that alter cell physiology by attuning ion currents across cellular and subcellular membranes. They are most commonly used to treat various noncommunicable diseases including diabetes and hypertension. While most ion transport modulators directly interact with ion transporters and ion channels, others including metformin indirectly alter ion transport by interfering with processes upstream to activation of the ion transporters and channels [10]. As the immunomodulatory activities of most ion transport modulators have not been fully characterised, we conducted this study to assess the immunomodulatory properties of thirteen FDA-approved ion transport modulators, namely, ambroxol $\mathrm{HCl}$, amiloride $\mathrm{HCl}$, diazoxide, digoxin, furosemide, hydrochlorothiazide (HCTZ), metformin, omeprazole, pantoprazole, phenytoin, verapamil, drug $\mathrm{X}$, and drug $\mathrm{Y}$. We recently reported that these compounds also have potent antimycobacterial activity [11]. We have withheld the identities of drug $\mathrm{X}$ and drug $\mathrm{Y}$ pending further studies.

\section{Materials and Methods}

2.1. Macrophages. THP-1, a human monocytic cell line, was obtained from American Type Culture Collection (ATCC) and cultured in RPMI 1640 (catalog no. R6504; SigmaAldrich, St. Louis, MO, USA) supplemented with 10\% FBS (catalog no. 30-2020; ATCC), $10 \mathrm{mM}$ HEPES (catalog no. H4034; Sigma-Aldrich), $4500 \mathrm{mg} / \mathrm{l}$ glucose (catalog no. G5500; Sigma-Aldrich), $1500 \mathrm{mg} / \mathrm{l}$ sodium bicarbonate (catalog no. S5761; Sigma-Aldrich), and $0.05 \mathrm{mM} \mathrm{2-}$ mercaptoethanol (catalog no. M6250; Sigma-Aldrich). Prior to each experiment, the THP-1 cells were differentiated into macrophages by treatment with $200 \mathrm{nM}$ phorbol-12-myristate-13-acetate (PMA) (catalog no. P8139; Sigma-Aldrich) for 3 days.

2.2. Mycobacterium bovis BCG. Mycobacterium bovis BCG-1 (Russia) was kindly donated by Citihealth Imports (Pvt) limited. It was grown in BD Difco Middlebrook $7 \mathrm{H} 9$ Broth (catalog no. DF0713-17-9, Thermo Fisher Scientific) supplemented with $10 \%(v / v)$ oleic acid-albumin-dextrose-catalase (OADC) growth supplement (catalog no. B12351, Thermo Fisher Scientific) and $0.05 \%$ tween 80 (catalog no. P4780; Sigma-Aldrich) to the mid-log phase. The bacteria were then pelleted by centrifugation at $3000 \mathrm{~g}$ for 15 minutes. The supernatant was decanted, and the pellet was resuspended in $70 \%$ ethanol and kept at room temperature for 2 hours. The bacteria were then centrifuged again at $3000 \mathrm{~g}$ for 15 minutes, and the pellet was washed twice with PBS. The pellet was then resuspended in PBS at a concentration of $3 \times 10^{6}$ $\mathrm{CFU} / \mathrm{ml}$, aliquoted, and stored at $-20^{\circ} \mathrm{C}$ until further use. To confirm that the $M$. bovis BCG had been inactivated, we plated it on BD Difco Middlebrook 7H10 media (catalog no. DF0627-17-4; Thermo Fisher Scientific) and observed no growth.

2.3. Drugs. All drugs that were tested in this study were purchased from Sigma-Aldrich. Stock solutions were prepared in
TABLE 1: Concentrations of drugs tested in the current study and their maximum free plasma concentrations in humans when taken at therapeutic doses.

\begin{tabular}{lcc}
\hline Drug & $\begin{array}{c}\text { Concentration used } \\
\text { in the current study } \\
\text { in ng/ml }\end{array}$ & $\begin{array}{c}\text { Maximum free } \\
\text { plasma concentration } \\
\text { in ng/ml }\end{array}$ \\
\hline Ambroxol HCl & 6 & $6[13]$ \\
Amiloride HCl & 40 & $40[14]$ \\
Diazoxide & 5000 & $5000[15]$ \\
Digoxin & 2 & $2[16]$ \\
Furosemide & 500 & $500[17]$ \\
HCTZ & 500 & $642[18]$ \\
Metformin & 1000 & $1000[19]$ \\
Omeprazole & 200 & $200[20]$ \\
Pantoprazole & 180 & $180[21]$ \\
Phenytoin & 2000 & $2000[22]$ \\
Verapamil & 24 & $24[23]$ \\
Drug X & 2 & 2 \\
Drug Y & 2 & 2 \\
\hline
\end{tabular}

Note. Reprinted from "Ion Transport Modulators as Antimycobacterial Agents," by SC Mitini-Nkhoma et al. 2020, Tuberculosis Research and Treatment, vol. 2020, doi:10.1155/2020/3767915.

dimethylsulfoxide (DMSO) (catalog no. C6295; SigmaAldrich), aliquoted, stored at $-20^{\circ} \mathrm{C}$, and used within six weeks. Working solutions were prepared in RPMI immediately before use. We assessed the toxicity of the drugs using the sulforhodamine B assay as previously described [12] and found that all the test drugs were not toxic to the macrophages at the concentrations that were used in this study (Table 1, and Figure S1 in Supplementary Materials). We used hydrocortisone (catalog no. H4001; Sigma-Aldrich), one of the most commonly used corticosteroids as a control in all the experiments, at a concentration of $75 \mathrm{ng} / \mathrm{ml}$.

2.4. Assessment of Effects of Ion Transport Modulators on Nitric Oxide Production. Nitric oxide (NO) is converted into nitrites $\left(\mathrm{NO}_{2}{ }^{-}\right)$and nitrates $\left(\mathrm{NO}_{3}{ }^{-}\right)$seconds after it is synthesized. $\mathrm{NO}_{2}{ }^{-}$and $\mathrm{NO}_{3}{ }^{-}$are therefore often used as surrogate measures for $\mathrm{NO}$ production [24]. We assessed total $\mathrm{NO}_{x}($ $\mathrm{NO}_{2}{ }^{-}+\mathrm{NO}_{3}{ }^{-}$) levels using the modified Griess assay as previously described, with slight modifications [25]. Griess reagent was prepared in-house by mixing equal volumes of $0.1 \% \mathrm{~N}$-(naphthyl)ethylenediamine (NED) (catalog no. 222488; Sigma-Aldrich) in distilled water with $1 \%$ sulfanilamide (catalog no. 46874; Sigma-Aldrich) in 5\% phosphoric acid (catalog no. W290017; Sigma-Aldrich) just before use.

We seeded $2 \times 10^{4}$ macrophages into each well of a 96well plate and stimulated them with $2 \times 10^{5} \mathrm{CFU}$ of ethanol-inactivated $M$. bovis BCG, in the presence or absence of each of the test drugs for 24 hours at $37^{\circ} \mathrm{C}$. The supernatant was then harvested and centrifuged at $5000 \mathrm{~g}$ for 10 minutes. Equal volumes of supernatant, Griess reagent, and vanadium (III) chloride (catalog no. 208272; Sigma-Aldrich) were mixed together and incubated for 30 minutes. We then measured the absorbance of the resulting azo dye at $540 \mathrm{~nm}$ using a multimode microplate reader $\left(\right.$ Synergy ${ }^{\mathrm{TM}}$ HTX, 
TABle 2: Primers used for quantification of cytokine RNA.

\begin{tabular}{|c|c|c|}
\hline Primer & Sequence $\left(5^{\prime}-3^{\prime}\right)$ & PrimerBank ID \\
\hline$\overline{\text { GAPDH (F) }}$ & ACAACTTTGGTATCGTGGAAGG & \multirow{2}{*}{$378404907 \mathrm{c} 2$} \\
\hline GAPDH (R) & GCCATCACGCCACAGTTTC & \\
\hline IL-1 $\beta(F)$ & ATGATGGCTTATTACAGTGGCAA & \multirow{2}{*}{$27894305 \mathrm{cl}$} \\
\hline IL- $1 \beta(\mathrm{R})$ & GTCGGAGATTCGTAGCTGGA & \\
\hline TNF- $\alpha(\mathrm{F})$ & ССТСТСТСТAATCAGCCCTCTG & \multirow{2}{*}{$25952110 \mathrm{c} 1$} \\
\hline TNF- $\alpha(\mathrm{R})$ & GAGGACCTGGGAGTAGATGAG & \\
\hline IL-10 (F) & GACTTTAAGGGTTACCTGGGTTG & \multirow{2}{*}{$24430216 c 1$} \\
\hline IL-10 (R) & TCACATGCGCCTTGATGTCTG & \\
\hline IL-12 $\alpha(\mathrm{F})$ & CCTTGCACTTCTGAAGAGATTGA & \multirow{2}{*}{$325974478 \mathrm{c} 1$} \\
\hline 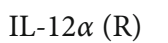 & ACAGGGCCATCATAAAAGAGGT & \\
\hline
\end{tabular}

BioTek, USA). The amount of $\mathrm{NO}_{x}$ produced in each culture was then interpolated from a standard curve generated using sodium nitrite.

2.5. Assessment of the Effect of Ion Transport Modulators on Superoxide Production. Superoxide production was assessed using the nitroblue tetrazolium (NBT) assay as previously described [26]. Superoxide reduces NBT to NBT-formazan, which can then be quantified spectrophotometrically to estimate the rate of superoxide production. We seeded $2 \times 10^{4}$ macrophages into each well of a 96-well plate in RPMI and stimulated them with $2 \times 10^{5} \mathrm{CFU}$ of ethanol-inactivated M. bovis BCG in the presence of NBT (catalog no. N6876, Sigma-Aldrich) and the test drugs over 30 minutes. The supernatant was then discarded, and the macrophages were washed twice with PBS. The macrophages, which now contained NBT-formazan, were fixed with 70\% methanol (cata$\log$ no. 179337, Sigma-Aldrich) and allowed to air dry. The NBT-formazan was solubilized in dissolving media containing dimethyl sulfoxide and potassium hydroxide (catalog no. P5958, Sigma-Aldrich). The suspension was centrifuged to remove cell debris, after which the amount of NBTformazan in the supernatant was quantified by measuring absorbance at $620 \mathrm{~nm}$ using a multimode microplate reader. The amount of NBT reduced in the drug-treated cultures was calculated as a percentage of that reduced by the drugfree controls as follows:

$$
\% \text { NBT reduction }=100 \times \frac{\text { OD test sample }}{\text { OD drug free control }} .
$$

2.6. Assessment of the Effects of Ion Transport Modulators on the Expression of Cytokine Genes. The expression of IL- $1 \beta$, IL-10, IL-12 $\alpha$, and TNF- $\alpha$ genes was assessed by RT-qPCR. We seeded $1 \times 10^{6}$ macrophages into each well of a 6 -well plate and stimulated them with $10 \times 10^{6} \mathrm{CFU}$ of ethanolinactivated $M$. bovis BCG in the presence or absence of the test drugs for 24 hours. RNA was then extracted using the RNEasy mini kit (Qiagen, Hilden, Germany), after which cDNA was synthesized using the GoScript reverse transcription system (Promega, Madison, USA). Quantitative PCR was performed using the Mesa green $\mathrm{qPCR}$ master mix
(Eurogentec, Seraing, Germany). All primer sequences were obtained from PrimerBank (Table 2), and the primers were synthesized by Integrated DNA Technologies (IDT).

2.7. Data Analysis. Data from the NBT and Griess assays were analysed using GraphPad Prism version 8.4.2 (GraphPad Software, La Jolla, California, USA). Production of nitric oxide and superoxide in the drug-treated samples and drug-free controls was compared using one-way analysis of variance (ANOVA) followed by Dunnett's post hoc test.

RNA expression data was analysed using $\mathrm{R}$ version 3.5.1 and the pcr library $[27,28]$. We used the Livak method to estimate the expression of the genes in the treated cultures relative to the drug-free controls [29], with GAPDH as a reference gene.

\section{Results and Discussion}

3.1. Ion Transport Modulators Alter Nitric Oxide Production in Macrophages. Nitric oxide production (indicated by total $\mathrm{NO}_{x}$ levels) was lower in macrophages that were treated with ambroxol $\mathrm{HCl}$, diazoxide, digoxin, furosemide, HCTZ, metformin, pantoprazole, phenytoin, verapamil, or drug Y than in drug-free controls $(p<0.001$, Figure 1). Macrophages that were treated with amiloride $\mathrm{HCl}$ produced more nitric oxide than drug-free controls $(p<0.001)$. There was no significant difference in nitric oxide production between drug-free controls and cultures that were treated with omeprazole or drug X.

Our findings are consistent with those of various authors, including Shen et al., who demonstrated that verapamil inhibits nitric oxide production in PMA-stimulated mouse peritoneal macrophages [30]. Verapamil inhibits the entry of calcium into cells through L-type voltage-gated calcium channels (VGCCs). Calcium is an important second messenger in several signal transduction pathways involved in macrophage activation [31]. Verapamil might therefore modulate nitric oxide production by interfering with calcium signalling.

Phenytoin and ambroxol $\mathrm{HCl}$ are potent inhibitors of voltage-gated sodium channels $\left(\mathrm{Na}_{\mathrm{V}} \mathrm{s}\right) \quad 1.5$ and 1.8, 


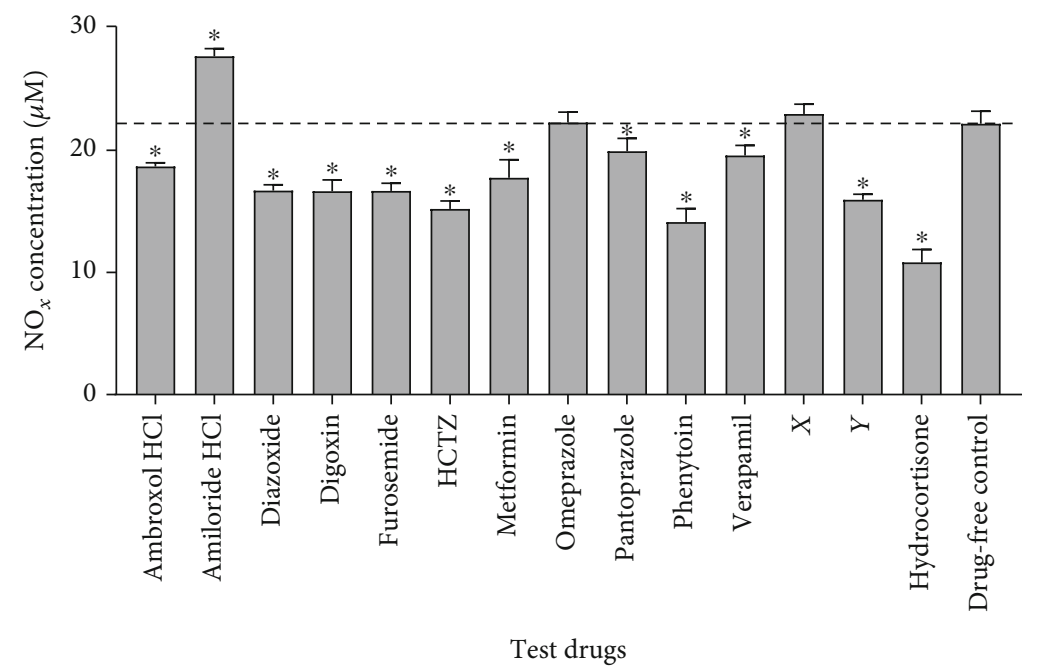

FIGURE 1: Effects of ion transport modulators on the production of nitrites and nitrates by THP-1-derived macrophages. Macrophages were stimulated with ethanol-inactivated M. bovis BCG in the presence or absence of the test drugs over 24 hours. Nitric oxide production was then assessed by quantifying $\mathrm{NO}_{x}$ levels in the supernatant using the Griess assay. Nitric oxide production was compared between the different cultures using one-way analysis of variance (ANOVA) followed by Dunnett's post hoc test. Data are presented as mean + SD of six replicates pooled from two independent experiments. Dashed line indicates nitric oxide production in drug-free controls. $*$ indicates $p<$ 0.05 vs. drug-free controls. HCTZ: hydrochlorothiazide.

respectively [32, 33]. Various authors have previously documented the inhibitory effects of both $\mathrm{Na}_{\mathrm{V}}$ inhibitors on nitric oxide production [34-36]. Phenytoin also inhibits various other macrophage functions including metalloproteinase production, which contributes to gingival hypertrophy in some people on chronic phenytoin therapy [37]. While the physiology of $\mathrm{Na}_{\mathrm{V}} 1.5$ has been most extensively studied in cardiac and neural tissue, Pappalardo et al. demonstrated that knockdown of $\mathrm{Na}_{\mathrm{V}} 1.5$ attenuates calcium influx in astrocytes [38]. Blocking $\mathrm{Na}_{\mathrm{V}} \mathrm{s}$ might therefore indirectly modulate leukocyte function by modulating calcium signalling.

Our findings also echo those of Kato et al., who demonstrated that metformin inhibits nitric oxide production in lipopolysaccharide- (LPS-) stimulated macrophages [39]. Interestingly, some studies have demonstrated that metformin enhances nitric oxide production, particularly in the absence of proinflammatory stimuli [40-42]. Nitric oxide production is mediated by 3 isoforms of the enzyme nitric oxide synthase (NOS), namely, neuronal NOS (nNOS), inducible NOS (iNOS), and endothelial NOS (eNOS) [43]. Of the three, nNOS and eNOS are generally expressed constitutively in various cell types, while the expression of iNOS is induced by proinflammatory stimuli [43]. It is therefore likely that metformin affects each isoform of NOS differently.

Our results also indicate that diazoxide inhibits nitric oxide production in macrophages. This is in agreement with the findings of Virgili et al., who demonstrated that diazoxide inhibits the production of nitric oxide in the murine model of multiple sclerosis [44]. Diazoxide is an agonist of ATP-gated potassium channels primarily used to treat patients with hypoglycaemia [15]. While calcium is more abundant in the extracellular fluid, potassium is highly concentrated in the cytosol. Opening of plasma membrane potassium channels leads to efflux of potassium down its chemical gradient, thus increasing the electrical gradient between the cytosol and the extracellular fluid. This increases the driving force for calcium influx. Diazoxide might therefore modulate nitric oxide production by indirectly modulating calcium signalling.

In the present study, macrophages that were treated with amiloride $\mathrm{HCl}$ produced more nitric oxide than drug-free controls. Amiloride $\mathrm{HCl}$ is primarily considered an inhibitor of $\mathrm{Na}+\mathrm{H}+$ exchangers (NHEs) but is also known to inhibit the activity of various sodium and calcium channels [45]. There is therefore a need for further studies to determine how amiloride $\mathrm{HCl}$ promotes nitric oxide production. While nitric oxide contributes to the pathogenesis of various diseases, it ameliorates pathology in others. Inhaled nitric oxide is used to treat various cardiopulmonary illnesses including acute respiratory distress syndrome [46]. Amiloride $\mathrm{HCl}$ and other agents which enhance nitric oxide production could potentially provide a cheaper and more convenient alternative to inhaled nitric oxide. Conversely, ambroxol $\mathrm{HCl}$, diazoxide, digoxin, furosemide, HCTZ, metformin, pantoprazole, phenytoin, verapamil, and drug Y, which demonstrated an inhibitory effect on the production of nitric oxide in this study, could potentially be used to ameliorate pathology in patients with septic shock and other diseases that involve anomalous production of nitric oxide.

3.2. Ion Transport Modulators Alter Superoxide Production by Macrophages. Superoxide production (indicated by \% NBT reduction) was lower in macrophages that were treated with any of the test drugs than in the drug-free controls (Figure 2). Macrophages that were treated with verapamil 


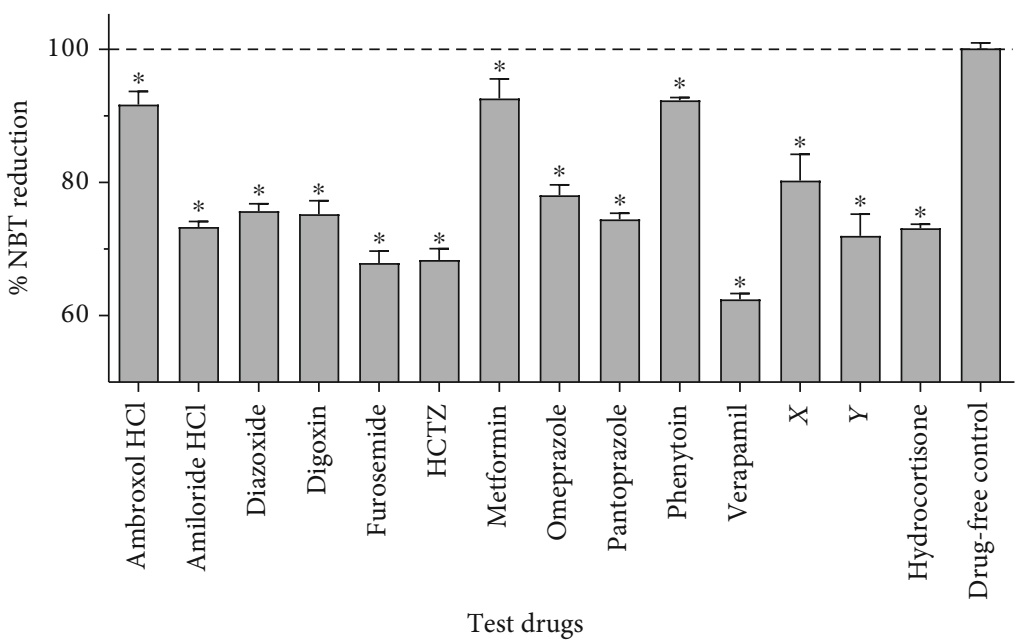

FIGURE 2: Effects of ion transport modulators on the production of superoxide by THP-1-derived macrophages. Macrophages were stimulated with ethanol-inactivated $M$. bovis BCG in the presence of the test drugs over 30 minutes, and production of superoxide was assessed using the NBT assay. Superoxide production was compared between the different cultures using one-way analysis of variance (ANOVA) followed by Dunnett's post hoc test. Data are presented as mean $(+\mathrm{SD})$ of six replicates pooled from two independent experiments. The dotted line indicates superoxide production in drug-free controls. $*$ indicates $p<0.05$ vs. drug-free controls. HCTZ: hydrochlorothiazide.

produced the least amount of superoxide $(62.4 \%, p<0.001)$. Our findings are in line with previous findings which have demonstrated that amiloride $\mathrm{HCl}$, ambroxol $\mathrm{HCl}$, metformin, omeprazole, and verapamil inhibit superoxide production by leukocytes [47-51]. Inhibition of superoxide production by the proton pump inhibitors (PPIs) omeprazole and pantoprazole, which are most commonly used to treat peptic ulcer disease and gastritis, might be one of the reasons for the high prevalence of gastrointestinal bacterial infections in people on chronic PPI therapy.

In macrophages, NADPH oxidases are the key producers of superoxide [52]. Shen et al. demonstrated that the calcium signalling pathway is involved in the activation of the NADPH oxidase [30]. As was the case with nitric oxide production, most of the compounds might have therefore inhibited superoxide production by indirectly modulating calcium signalling. In addition to NADPH oxidases, the three isoforms of NOS also contribute to superoxide production in macrophages [53]. Therefore, the test drugs also might have reduced nitric oxide production in the macrophages by inhibiting the activity of iNOS.

Hydrocortisone is one of the most commonly used corticosteroids in clinical practice. In the present study, superoxide production was lower in macrophages that were treated with furosemide, HCTZ, verapamil, or drug Y than macrophages that were treated with hydrocortisone. Unlike ion transport modulators, hydrocortisone primarily functions by altering cellular transcription patterns and thus has a slow onset [3]. The effect of hydrocortisone on the macrophages was therefore likely not maximal at the time the experiment was completed.

As superoxide is implicated in the pathogenesis of various diseases including atherosclerosis, myocardial infarction, and cerebrovascular accidents [54], ion channel modulators could potentially be used to ameliorate pathology in such diseases.
3.3. Ion Transport Modulators Alter the Expression of Cytokines by Macrophages. Macrophages that were treated with amiloride $\mathrm{HCl}$, diazoxide, digoxin, furosemide, phenytoin, verapamil drug $\mathrm{X}$, and drug $\mathrm{Y}$ had higher levels of expression of TNF- $\alpha$ and IL- $1 \beta$ genes than drug-free controls $(p<0.001$, Figure 3$)$. Macrophages that were treated with metformin and pantoprazole had higher levels of expression of TNF- $\alpha$ but not IL- $1 \beta$. The expression of TNF- $\alpha$ was highest in macrophages that were treated with furosemide, while that of IL- $1 \beta$ was highest in macrophages that were treated with phenytoin. There was no statistically significant difference in the expression of TNF- $\alpha$ and IL-1 $\beta$ between cultures that were treated with ambroxol $\mathrm{HCl}$ or omeprazole and the drug-free controls.

We did not observe any statistically significant differences in the expression of IL-12 $\alpha$ and IL-10 between the drug-free controls and any of the test conditions.

Our findings are in line with those of Gupta et al. who demonstrated that inhibiting the activity of L-type voltagegated calcium channels enhances the expression of proinflammatory cytokines in macrophages and dendritic cells stimulated with Mycobacterium tuberculosis (Mtb) lysate [55]. However, Li et al. observed contrasting findings following concomitant exposure of Sprague-Dawley rats to LPS and verapamil [56]. The incongruity is likely because different stimulants invoke different combinations of cell activation pathways, leading to differences in cellular responses to both the stimulants and any concomitantly administered compounds. In addition, different cell types may respond differently to both the stimulants and the ion transport modulators.

As with verapamil, there is contrasting literature on the effects of the sodium channel agonists phenytoin and ambroxol $\mathrm{HCl}$, and indeed other ion channel modulators on cytokine production by macrophages. For example, while 


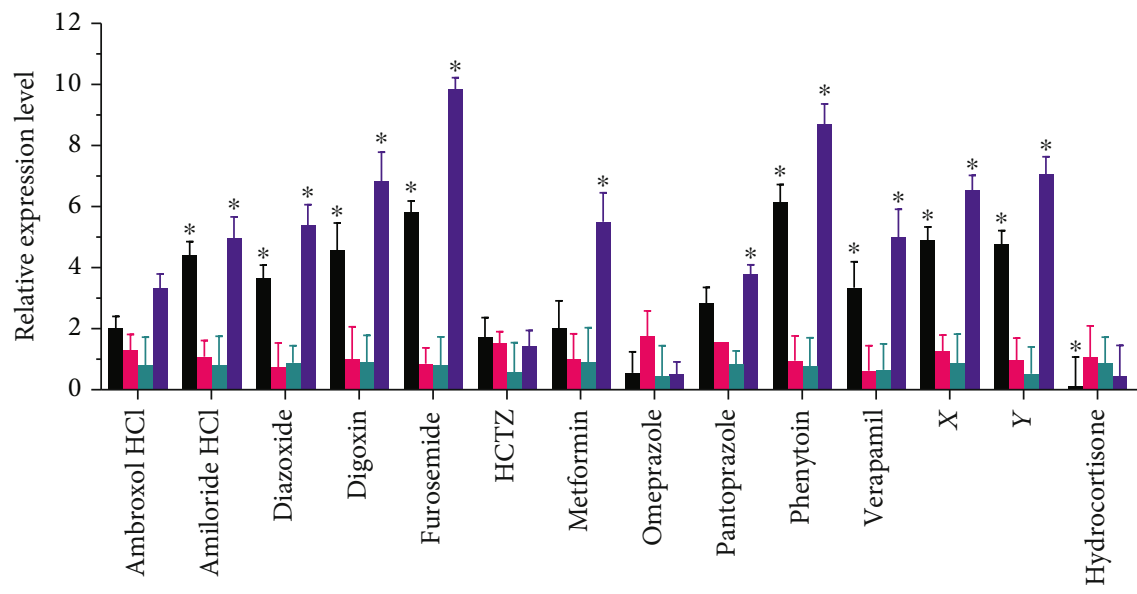

Test drugs

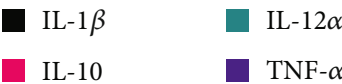

FIGURE 3: Effect of ion transport modulators on cytokine expression by macrophages. Macrophages were stimulated with ethanol-inactivated $M$. bovis BCG in the presence of the test drugs over 24 hours. RNA expression levels in the drug-treated cultures relative to the drug-free controls were calculated using the Livak method. Data are presented as mean $(+\mathrm{SD})$ of two replicates. $*$ indicates $p<0.05$ vs. drug-free controls. HCTZ: hydrochlorothiazide.

TABLE 3: Summary of immunomodulatory activity of ion transport modulators.

\begin{tabular}{|c|c|c|c|c|c|c|}
\hline & Nitric oxide & Superoxide & TNF- $\alpha$ & IL- $1 \beta$ & IL-10 & IL-12 $\alpha$ \\
\hline Ambroxol $\mathrm{HCl}$ & $\downarrow$ & $\downarrow$ & $\leftrightarrow$ & $\leftrightarrow$ & $\leftrightarrow$ & $\leftrightarrow$ \\
\hline Amiloride $\mathrm{HCl}$ & $\uparrow$ & $\downarrow$ & $\uparrow$ & $\uparrow$ & $\leftrightarrow$ & $\leftrightarrow$ \\
\hline Diazoxide & $\downarrow$ & $\downarrow$ & $\uparrow$ & $\uparrow$ & $\leftrightarrow$ & $\leftrightarrow$ \\
\hline Digoxin & $\downarrow$ & $\downarrow$ & $\uparrow$ & $\uparrow$ & $\leftrightarrow$ & $\leftrightarrow$ \\
\hline Furosemide & $\downarrow$ & $\downarrow$ & $\uparrow$ & $\uparrow$ & $\leftrightarrow$ & $\leftrightarrow$ \\
\hline HCTZ & $\downarrow$ & $\downarrow$ & $\leftrightarrow$ & $\leftrightarrow$ & $\leftrightarrow$ & $\leftrightarrow$ \\
\hline Metformin & $\downarrow$ & $\downarrow$ & $\uparrow$ & $\leftrightarrow$ & $\leftrightarrow$ & $\leftrightarrow$ \\
\hline Omeprazole & $\leftrightarrow$ & $\downarrow$ & $\leftrightarrow$ & $\leftrightarrow$ & $\leftrightarrow$ & $\leftrightarrow$ \\
\hline Pantoprazole & $\downarrow$ & $\downarrow$ & $\uparrow$ & $\leftrightarrow$ & $\leftrightarrow$ & $\leftrightarrow$ \\
\hline Phenytoin & $\downarrow$ & $\downarrow$ & $\uparrow$ & $\uparrow$ & $\leftrightarrow$ & $\leftrightarrow$ \\
\hline Verapamil & $\downarrow$ & $\downarrow$ & $\uparrow$ & $\uparrow$ & $\leftrightarrow$ & $\leftrightarrow$ \\
\hline $\mathrm{X}$ & $\leftrightarrow$ & $\downarrow$ & $\uparrow$ & $\uparrow$ & $\leftrightarrow$ & $\leftrightarrow$ \\
\hline $\mathrm{Y}$ & $\downarrow$ & $\downarrow$ & $\uparrow$ & $\uparrow$ & $\leftrightarrow$ & $\leftrightarrow$ \\
\hline
\end{tabular}

Song and Cheng demonstrated that phenytoin promotes production of TNF- $\alpha$ by wound macrophages following systemic and local irradiation in mice [57], Jackson et al. observed that phenytoin inhibits TNF- $\alpha$ production in human multipotent adult progenitor cells [58]. In addition, Ichiyama et al. reported that phenytoin has no effect on the production of TNF- $\alpha$ in LPS-stimulated THP-1 cells [59]. The incongruency in the results obtained from different systems therefore indicates that ion transport modulators do not cause a global depression of inflammatory processes and may enhance others depending on the context.

3.4. Relationship between Nitric Oxide Production, Superoxide Production, and Cytokine Expression. Table 3 summarises the immunomodulatory activities of the ion transport modulators tested in this study.

We pooled together the data obtained with all the test drugs in all the assays to assess the correlation between the different aspects of macrophage function (Figure 4). We observed a strong positive correlation between TNF- $\alpha$ and IL-1 $\beta$ expression ( $r=0.92, p \leq 0.001$, Figure 4$)$, and a strong negative correlation between the expression of IL-10 and TNF- $\alpha(r=-0.7, p=0.007)$. We also observed a negative albeit weak correlation between the production of nitric oxide and the expression of TNF- $\alpha(r=-0.31, p=0.308)$. This finding is consistent with that of Thomassen et al. who demonstrated that nitric oxide downregulates the production of IL-1 $\beta$ and TNF- $\alpha$ in human alveolar macrophage [60]. 


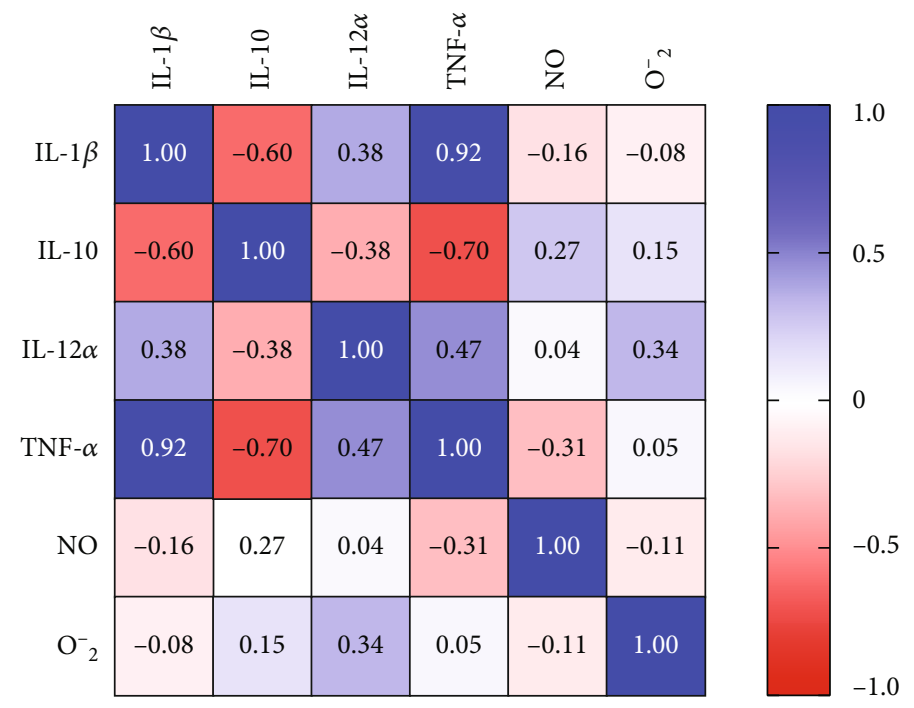

FIGURE 4: Spearman correlation coefficients between different markers of macrophage function.

Therefore, the test compounds might have enhanced the expression of TNF- $\alpha$ and IL- $1 \beta$ by inhibiting nitric oxide production, thus removing the tonic inhibition on the expression of proinflammatory cytokines.

\section{Conclusions}

In summary, we have demonstrated that ambroxol $\mathrm{HCl}$, amiloride $\mathrm{HCl}$, diazoxide, digoxin, furosemide, HCTZ, metformin, omeprazole, pantoprazole, phenytoin, verapamil, drug $\mathrm{X}$, and drug $\mathrm{Y}$ have potent immunomodulatory properties. More importantly, the results indicate that the ion transport modulators differentially alter various aspects of macrophage function, unlike most immunomodulators in clinical practice which depress all immune responses. Therefore, ion transport modulators could potentially serve as more selective immunomodulators, with a lower risk of causing overt immunosuppression.

4.1. Limitations. While we assessed the expression of cytokine genes, we could not assess their secretion. In addition, as this study was conducted in vitro, it might not accurately reflect how the ion channel modulators would affect macrophage physiology in vivo. There is therefore a need to ascertain the immunomodulatory properties of the ion transport modulators tested in this study in in vivo systems.

\section{Data Availability}

The data used to support the findings of this study are available from the authors upon request.

\section{Disclosure}

The current address of G.K.D. Ishaka is Australian College of Business and Technology, 442 Galle Road, Colombo 3, Sri Lanka.

\section{Conflicts of Interest}

The authors declare that there is no conflict of interest regarding the publication of this paper.

\section{Acknowledgments}

This work was funded by the Association of Commonwealth Universities (ACU) and the Institute of Biochemistry, Molecular Biology and Biotechnology, University of Colombo.

\section{Supplementary Materials}

Figure S1: mean (+SD) viability of THP-1-derived macrophages following exposure to different concentrations of test drugs. (Supplementary Materials)

\section{References}

[1] G. S. Cooper, M. L. K. Bynum, and E. C. Somers, "Recent insights in the epidemiology of autoimmune diseases: improved prevalence estimates and understanding of clustering of diseases," Journal of Autoimmunity, vol. 33, no. 3-4, pp. 197-207, 2009.

[2] C. W. Schmidt, "Questions persist: environmental factors in autoimmune disease," Environmental Health Perspectives, vol. 119, no. 6, pp. A248-A253, 2011.

[3] S. Ramamoorthy and J. A. Cidlowski, "Corticosteroids: mechanisms of action in health and disease," Rheumatic Disease Clinics of North America, vol. 42, no. 1, pp. 15-31, 2016.

[4] C. Wang, Y. Liu, and J. M. Cao, "G protein-coupled receptors: extranuclear mediators for the non-genomic actions of steroids," International Journal of Molecular Sciences, vol. 15, no. 9, pp. 15412-15425, 2014.

[5] W. Ericson-Neilsen and A. D. Kaye, "Steroids: pharmacology, complications, and practice delivery issues," The Ochsner Journal, vol. 14, no. 2, pp. 203-207, 2014. 
[6] J. R. Vane, "Inhibition of prostaglandin synthesis as a mechanism of action for aspirin-like drugs," Nature New Biology, vol. 231, no. 25, pp. 232-235, 1971.

[7] A. Zarghi and S. Arfaei, "Selective COX-2 inhibitors: a review of their structure-activity relationships," Iranian Journal of Pharmaceutical Research, vol. 10, no. 4, pp. 655-683, 2011.

[8] S. Wongrakpanich, A. Wongrakpanich, K. Melhado, and J. Rangaswami, "A comprehensive review of non-steroidal anti-inflammatory drug use in the elderly," Aging and Disease, vol. 9, no. 1, pp. 143-150, 2018.

[9] R. J. Bing and M. Lomnicka, "Why do cyclo-oxygenase-2 inhibitors cause cardiovascular events?," Journal of the American College of Cardiology, vol. 39, no. 3, pp. 521-522, 2002.

[10] X. Fu, Y. Pan, Q. Cao et al., "Metformin restores electrophysiology of small conductance calcium-activated potassium channels in the atrium of GK diabetic rats," BMC Cardiovascular Disorders, vol. 18, no. 1, p. 63, 2018.

[11] S. C. Mitini-Nkhoma, N. Fernando, G. K. D. Ishaka, S. M. Handunnetti, and S. L. Pathirana, "Ion transport modulators as antimycobacterial agents," Tuberculosis Research and Treatment, vol. 2020, 7 pages, 2020.

[12] S. R. Samarakoon, I. Thabrew, P. Galhena, D. De Silva, and K. Tennekoon, "A comparison of the cytotoxic potential of standardized aqueous and ethanolic extracts of a polyherbal mixture comprised of Nigella sativa (seeds), Hemidesmus indicus (roots) and Smilax glabra (rhizome)," Pharmacognosy Research, vol. 2, no. 6, pp. 335-342, 2010.

[13] Y. G. Yang, L. X. Song, N. Jiang, X. T. Xu, and Z. M. Di XH, "Pharmacokinetics of ambroxol and clenbuterol tablets in healthy Chinese volunteers," International Journal of Clinical and Experimental Medicine, vol. 8, pp. 18744-18750, 2015.

[14] K. M. Jones, E. Liao, K. Hohneker et al., "Pharmacokinetics of amiloride after inhalation and oral administration in adolescents and adults with cystic fibrosis," Pharmacotherapy, vol. 17, no. 2, pp. 263-270, 1997.

[15] R. Kizu, K. Nishimura, R. Sato et al., "Population pharmacokinetics of diazoxide in children with hyperinsulinemic hypoglycemia," Hormone Research in Paediatrics, vol. 88, no. 5, pp. 316-323, 2017.

[16] T. W. Smith, V. P. Butler, and E. Haber, "Determination of therapeutic and toxic serum digoxin concentrations by radioimmunoassay," The New England Journal of Medicine, vol. 281, no. 22, pp. 1212-1216, 1969.

[17] University of Lausanne, "Furosemide," 2018, https://sepia2 .unil.ch/pharmacology/index.php?id=102. June 7, 2019.

[18] R. Aubin, P. Ménard, and D. Lajeunesse, "Selective effect of thiazides on the human osteoblast-like cell line MG-63," Kidney International, vol. 50, no. 5, pp. 1476-1482, 1996.

[19] F. Kajbaf, M. E. De Broe, and J.-D. Lalau, "Therapeutic concentrations of metformin: a systematic review," Clinical Pharmacokinetics, vol. 55, no. 4, pp. 439-459, 2016.

[20] J. M. Shin and N. Kim, "Pharmacokinetics and pharmacodynamics of the proton pump inhibitors," Journal of Neurogastroenterology and Motility, vol. 19, no. 1, pp. 25-35, 2013.

[21] R. Huber, M. Hartmann, H. Bliesath, R. Lühmann, V. W. Steinijans, and K. Zech, "Pharmacokinetics of pantoprazole in man," International Journal of Clinical Pharmacology and Therapeutics, vol. 34, 1 Suppl, pp. S7-16, 1996.

[22] J. Galjour, Phenytoin Level, Medscape, 2014, https://emedicine .medscape.com/article/2090306-overview\#a1. June 7, 2019.
[23] W. Frishman, E. Kirsten, M. Klein et al., "Clinical relevance of verapamil plasma levels in stable angina pectoris," The American Journal of Cardiology, vol. 50, no. 5, pp. 1180-1184, 1982.

[24] N. S. Bryan and M. B. Grisham, "Methods to detect nitric oxide and its metabolites in biological samples," Free Radical Biology \& Medicine, vol. 43, no. 5, pp. 645-657, 2007.

[25] G. C. Park, J. S. Ryu, and D. Y. Min, “The role of nitric oxide as an effector of macrophage-mediated cytotoxicity against Trichomonas vaginalis," The Korean Journal of Parasitology, vol. 35, no. 3, pp. 189-195, 1997.

[26] H. Sim Choi, J. Woo Kim, Y.-N. Cha, and C. Kim, "A quantitative nitroblue tetrazolium assay for determining intracellular superoxide anion production in phagocytic cells," Journal of Immunoassay \& Immunochemistry, vol. 27, no. 1, pp. 31-44, 2006.

[27] R Core Team, R: A Language and Environment for Statistical Computing, R Foundation for Statistical Computing, Vienna, Austria, 2018.

[28] M. Ahmed and D. R. Kim, "pcr: an R package for quality assessment, analysis and testing of qPCR data," PeerJ, vol. 6, article e4473, 2018.

[29] K. J. Livak and T. D. Schmittgen, "Analysis of relative gene expression data using real-time quantitative PCR and the 2$\Delta \Delta$ CT method," Methods, vol. 25, no. 4, pp. 402-408, 2001.

[30] H. Shen, M. D. Wiederhold, and D. W. Ou, "The suppression of macrophage secretion by calcium blockers and adenosine," Immunopharmacology and Immunotoxicology, vol. 17, no. 2, pp. 301-309, 1995.

[31] A. C. Newton, M. D. Bootman, and J. Scott, "Second messengers," Cold Spring Harbor Perspectives in Biology, vol. 8, no. 8, article a005926, 2016.

[32] W. Gaida, K. Klinder, K. Arndt, and T. Weiser, "Ambroxol, a Nav1.8-preferring $\mathrm{Na}+$ channel blocker, effectively suppresses pain symptoms in animal models of chronic, neuropathic and inflammatory pain," Neuropharmacology, vol. 49, no. 8, pp. 1220-1227, 2005.

[33] M. Yang, D. J. Kozminski, L. A. Wold et al., "Therapeutic potential for phenytoin: targeting $\mathrm{Na}_{\mathrm{v}} 1.5$ sodium channels to reduce migration and invasion in metastatic breast cancer," Breast Cancer Research and Treatment, vol. 134, no. 2, pp. 603-615, 2012.

[34] L. P. Reagan, C. R. McKittrick, and B. S. McEwen, "Corticosterone and phenytoin reduce neuronal nitric oxide synthase messenger RNA expression in rat hippocampus," Neuroscience, vol. 91, no. 1, pp. 211-219, 1999.

[35] Y. Y. Jang, J. H. Song, Y. K. Shin, E. S. Han, and C. S. Lee, "Depressant effects of ambroxol and erdosteine on cytokine synthesis, granule enzyme release, and free radical production in rat alveolar macrophages activated by lipopolysaccharide," Pharmacology \& Toxicology, vol. 92, no. 4, pp. 173-179, 2003.

[36] F. L. M. Ricciardolo, V. Sorbello, S. Benedetto, and D. Paleari, "Effect of ambroxol and beclomethasone on lipopolysaccharide-induced nitrosative stress in bronchial epithelial cells," Respiration, vol. 89, no. 6, pp. 572-582, 2015.

[37] J. D. Corrêa, C. M. Queiroz-Junior, J. E. Costa, A. L. Teixeira, and T. A. Silva, "Phenytoin-induced gingival overgrowth: a review of the molecular, immune, and inflammatory features," ISRN Dentistry, vol. 2011, 8 pages, 2011.

[38] L. W. Pappalardo, O. A. Samad, J. A. Black, and S. G. Waxman, "Voltage-gated sodium channel $\mathrm{Na}_{\mathrm{v}} 1.5$ contributes to 
astrogliosis in an in vitro model of glial injury via reverse $\mathrm{Na}^{+} / \mathrm{Ca}^{2+}$ exchange," Glia, vol. 62, no. 7, pp. 1162-1175, 2014.

[39] Y. Kato, N. Koide, T. Komatsu et al., "Metformin attenuates production of nitric oxide in response to lipopolysaccharide by inhibiting MyD88-independent pathway," Hormone and Metabolic Research, vol. 42, no. 9, pp. 632-636, 2010.

[40] B. J. Davis, Z. Xie, B. Viollet, and M. H. Zou, "Activation of the AMP-activated kinase by antidiabetes drug metformin stimulates nitric oxide synthesis in vivo by promoting the association of heat shock protein 90 and endothelial nitric oxide synthase," Diabetes, vol. 55, no. 2, pp. 496-505, 2006.

[41] Y. W. Kim, S. Y. Park, J. Y. Kim et al., "Metformin restores the penile expression of nitric oxide synthase in high-fat-fed obese rats," Journal of Andrology, vol. 28, no. 4, pp. 555-560, 2007.

[42] T. R. O'Hora, F. Markos, N. F. Wiernsperger, and M. I. M. Noble, "Metformin causes nitric oxide-mediated dilatation in a shorter time than insulin in the iliac artery of the anesthetized pig," Journal of Cardiovascular Pharmacology, vol. 59, no. 2, pp. 182-187, 2012.

[43] U. Förstermann and W. C. Sessa, "Nitric oxide synthases: regulation and function," European Heart Journal, vol. 33, no. 7, pp. 829-837, 2012.

[44] N. Virgili, J. F. Espinosa-Parrilla, P. Mancera et al., "Oral administration of the KATP channel opener diazoxide ameliorates disease progression in a murine model of multiple sclerosis," Journal of Neuroinflammation, vol. 8, no. 1, p. 149, 2011.

[45] M. L. Garcia, V. F. King, J. L. Shevell et al., "Amiloride analogs inhibit L-type calcium channels and display calcium entry blocker activity.," Journal of Biological Chemistry, vol. 265, no. 7, pp. 3763-3771, 1990.

[46] J. Á. Monsalve-Naharro, E. Domingo-Chiva, S. García Castillo, P. Cuesta-Montero, and J. M. Jiménez-Vizuete, "Inhaled nitric oxide in adult patients with acute respiratory distress syndrome," Farmacia Hospitalaria, vol. 41, no. 2, pp. 292-312, 2017.

[47] J. H. Wandall, "Effects of omeprazole on neutrophil chemotaxis, super oxide production, degranulation, and translocation of cytochrome b-245," Gut, vol. 33, no. 5, pp. 617-621, 1992.

[48] F. Khalfi, B. Gressier, T. Dine et al., "Verapamil inhibits elastase release and superoxide anion production in human neutrophils," Biological \& Pharmaceutical Bulletin, vol. 21, no. 2, pp. 109-112, 1998.

[49] M. Suzuki, S. Teramoto, T. Matsuse et al., "Inhibitory effect of ambroxol on superoxide anion production and generation by murine lung alveolar macrophages," The Journal of Asthma, vol. 35, no. 3, pp. 267-272, 1998.

[50] Ł. Bułdak, K. Łabuzek, R. J. Bułdak et al., "Metformin affects macrophages' phenotype and improves the activity of glutathione peroxidase, superoxide dismutase, catalase and decreases malondialdehyde concentration in a partially AMPKindependent manner in LPS- stimulated human monocytes/macrophages," Pharmacological Reports, vol. 66, no. 3, pp. 418-429, 2014.

[51] M. W. Verghese and R. C. Boucher, "Effects of ion composition and tonicity on human neutrophil antibacterial activity," American Journal of Respiratory Cell and Molecular Biology, vol. 19, no. 6, pp. 920-928, 1998.

[52] Y. Groemping and K. Rittinger, "Activation and assembly of the NADPH oxidase: a structural perspective," The Biochemical Journal, vol. 386, no. 3, pp. 401-416, 2005.
[53] Y. Xia, "Superoxide generation from nitric oxide synthases," Antioxidants \& Redox Signaling, vol. 9, no. 10, pp. 17731778, 2007.

[54] J. Frostegård, "Immunity, atherosclerosis and cardiovascular disease," BMC Medicine, vol. 11, no. 1, article 117, 2013.

[55] S. Gupta, N. Salam, V. Srivastava et al., "Voltage gated calcium channels negatively regulate protective immunity to Mycobacterium tuberculosis," PLoS One, vol. 4, no. 4, article e5305, 2009.

[56] G. Li, X. P. Qi, X. Y. Wu et al., "Verapamil modulates LPSinduced cytokine production via inhibition of NF-kappa B activation in the liver," Inflammation Research, vol. 55, no. 3, pp. 108-113, 2006.

[57] S. Song and T. Cheng, "The effect of systemic and local irradiation on wound macrophages and the repair promoting action of phenytoin sodium," Zhonghua Yi Xue Za Zhi, vol. 77, pp. 54-57, 1997.

[58] M. L. Jackson, K. A. Ruppert, D. J. Kota et al., "Clinical parameters affecting multipotent adult progenitor cells in vitro," Heliyon, vol. 5, no. 10, article e02532, 2019.

[59] T. Ichiyama, K. Okada, J. M. Lipton, T. Matsubara, T. Hayashi, and S. Furukawa, "Sodium valproate inhibits production of TNF-alpha and IL-6 and activation of NF- $\kappa \mathrm{B}$," Brain Research, vol. 857, no. 1-2, pp. 246-251, 2000.

[60] M. J. Thomassen, L. T. Buhrow, M. J. Connors, F. T. Kaneko, S. C. Erzurum, and M. S. Kavuru, "Nitric oxide inhibits inflammatory cytokine production by human alveolar macrophages," American Journal of Respiratory Cell and Molecular Biology, vol. 17, no. 3, pp. 279-283, 1997. 